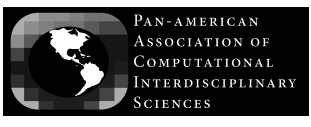

Journal of Computational Interdisciplinary Sciences (2009) 1(2): 83-88

(C) 2009 Pan-American Association of Computational Interdisciplinary Sciences

ISSN 1983-8409

http://epacis.org

\title{
A model for patchy stomatal conductance in homobaric leaves
}

\author{
Marcus C. Ferraz ${ }^{1}$, Gustavo M. Souza ${ }^{2}$ and Carmen P.C. Prado ${ }^{1}$ \\ Manuscript received on October 28, 2008 / accepted on January 20, 2009
}

\begin{abstract}
Many studies in the last years showed that, for a wide variety of plants, stomata apertures exhibits spatial temporal patterns in a phenomena known as patchy stomatal conductance. Many attempts were made to build numerical models to explain this complex behavior, but none were able to explain all of behaviors observed in nature. In this work we discuss some limitations of an hydraulic cellular automaton model proposed by Haefner, Buckley \& Mott in 1997 (PC\&E,20,1997). Besides considering a more realistic geometry, in which the areola are not hydraulically isolated, as in the original model, we show that small changes in the dynamics of individual stomata can lead to patchy behavior.
\end{abstract}

Keywords: mathematical modeling, non-linear dynamics, patchy stomatal conductance, plant gas exchange, hysteresis. 


\section{INTRODUCTION}

Stomata play an important role in the physiology of plants. They perform a control task, allowing necessary carbon assimilation while preventing excessive water loss. Most of the water absorbed by a plant is lost through stomata and practically all $\mathrm{CO}_{2}$ entering the plant also passes through them. Plants can control and adjust stomata apertures in order to adapt themselves to changes in environmental conditions.

Until some time ago, experiments on stomatal conductance could measure only the average behavior of the leaf, and it was assumed that individual stomata reacted independently one from the other to external stimuli, with a normal distribution of apertures $[13,16]$. However, with the development of more precise experimental techniques, as chlorophyll fluorescence, it was possible to measure the behavior of stomata in smaller regions of the leaf, showing that this assumption was not correct. In a large number of plants, under some circumstances like hydraulic stress, stomata displayed a rich dynamics with the appearance of complex spatio-temporal patterns, in a behavior known as patchy stomatal conductance $[7,8,10,14,15]$. The origin of this complex behavior is still an open question. One of the possible hypotheses is that this patchy behavior is a signature of the complex mechanisms governing water transport in the mesophyll of the leaf.

In this paper we explore the possible role played by mechanisms of water transport in the mesophyll in the onset of patchiness, and examine some properties of the dynamics of individual stomata. Some attempts to explain patchiness by modeling hydraulic interactions among stomata have already been considered $[5,7,10]$. In our opinion, however, these attempts were not able to generate the wealth of behavior observed in nature, as claimed. We show that a more realistic, less restrictive geometry as well as memory effects in the dynamics of each pore are enough to give rise to a very rich collective response. Our model is more adequate to describe homobaric leaves, in which areolas are not isolated one from the other [11]. In the following sections we revisit the pioneering work of [5], on which we based most of our own work, presenting our criticism to their findings. Then, we present and discuss some modifications to their model, in particular to the geometry of the model and the dynamic behavior of each pore. Finally, we summarize our findings in the conclusions.

\section{THE HYDRAULIC HYPOTHESIS}

Some models for single stoma dynamics, based on hydraulic considerations [4, 12], are able to predict different dynamic responses, but contain a large number of parameters. That fact limits their use in understanding the dominant mechanisms responsible for the onset of patchiness. Also, these models are not adequate to describe any collective behavior (patchiness). More recently, [5] proposed a two-dimensional hydraulic model, taking into account interactions between neighboring stomata, which are supposed to be arranged in a very regular and restrictive geometry. In their model the leaf is represented by a square grid; each site of this grid is a stomatal unit (SU), consisting of a pair of guard cells and the surrounding epidermal tissue. Interactions between guard cells and the epidermal tissue, as well as between each stomatal unit and its neighbors, are taken into account. Each subset of $4 \times 4$ SUs form an areola, and areolas are separated one from another by veins, that act as water reservoirs (see Fig. 1).

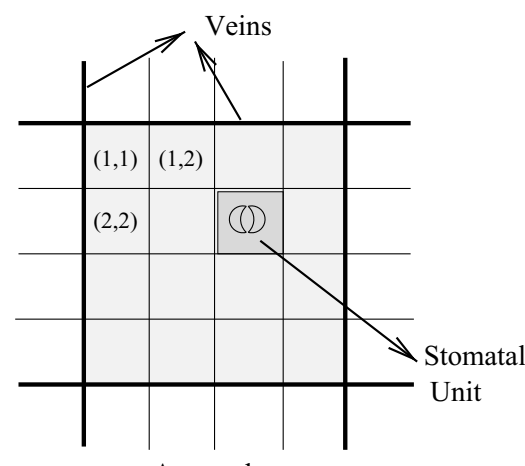

An areola

Figure 1 - Geometry of the model proposed by Haefner, Buckley and Mott in 1997. An areola was made of $4 \times 4$ Stomatal Units and completely surrounded by veins, for which the water potential was kept constant.

The dynamics of this system is governed by a set of discrete rules, which were inspired in differential equations associated with accepted biological hypotheses. They also assumed a piecewise linear, slow and continuous function to describe the relationship between stomata apertures and the turgor pressure differences between guard cells and the epidermis. Our work employs the same equations, except for this last one.

Two independent variables are associated to each SU of the grid: the water potential $\Psi$ of the whole SU and the osmotic pressure $\pi_{g}$ of guard cells. Due to close contact and experimental evidences, there is no distinction between the water potentials of guard cells and the surrounding epidermis, so they were considered the same. The osmotic pressure $\pi_{e}$ of epidermis is constant. The water potential $\Psi$ of a generic stomatal unit $i$ evolves in time according to

$$
\Psi^{i}(t+1)=\Psi^{i}(t)+a\left[F^{i}(t)-E^{i}(t)\right],
$$

where $F^{i}=\sum_{j=1}^{n}\left(\Psi^{j}-\Psi^{i}\right)$ is the flow to site $i$ (the sum is performed over all $n=8$ neighbors). The evaporation rate 
$E^{i}$ is proportional to the water vapor mole fraction deficit $\Delta w$ and to the stomata aperture $A^{i}$,

$$
E^{i}=q \Delta w A^{i}
$$

The aperture $A^{i}$ of the stomata depends on the difference in turgor pressures $P_{g}$ and $P_{e}$ of the guard cells and the surrounding epidermis ( $P_{e, g}=\Psi-\pi_{e, g}$, if $\pi_{e, g}<\Psi$, zero otherwise)

$$
A^{i}= \begin{cases}P_{g}^{i}-\theta^{i} P_{e}^{i} & \text { if } P_{g}^{i}>\theta^{i} P_{e}^{i} \\ 0 & \text { otherwise }\end{cases}
$$

$\theta^{i}$ is the rate between mechanical coefficients of guard and epidemic cells, and can vary slightly for each SU. $A^{i}$ grows linearly with $P_{g}-P_{e}$ except for a threshold defined by $\theta^{i}$. The conductance $g^{i}$, that is usually measured in experiments, is proportional to the aperture $A^{i}$. The osmotic pressure $\pi_{g}^{i}$ of a guard cell in stomatal unit $i$ evolves in time towards a maximum value $\pi^{i, s s}(t)$, at a rate proportional to the difference between the current value of $\pi_{g}^{i}$ and $\pi^{i, s s}(t)$,

$$
\pi_{g}^{i}(t+1)=\pi_{g}^{i}(t)+\alpha\left(\pi^{i, s s}(t)-\pi_{g}^{i}(t)\right) .
$$

The maximum value $\pi^{i, s s}$ changes with site and time, according to the epidermal turgor pressure $P_{e}$,

$$
\pi^{i, s s}(t)=\pi_{g}^{\min }+\frac{P_{e}^{i}(t)}{P_{e}^{\max }}\left(\pi_{g}^{\max }-\pi_{g}^{\min }\right),
$$

where $P_{e}^{\max }, \pi_{e}^{\max }$ and $\pi_{e}^{\min }$ are parameters of the model. The water potential $\Psi$ of the veins was kept constant and equal to zero. Equations (1) to (5) are iterated to give the time evolution of the system.

We carefully tried to reproduce the results of Haefner et al. [5]. Except for the values of $a$ and $\alpha$, we simulated the above equations with the same parameter values employed in their work. We also built a graphic interface, placing in each cell (SU) a black circle with a diameter proportional to the aperture of the stomata. The visualization of the process confirmed some of the results presented in this paper. We noticed also that there were no qualitative differences in assuming a triangular distribution for mechanical coefficients $\theta^{i}$ (as employed in [5]), and an uniform distribution between a minimum and maximum values. For simplicity, then, we adopted the latter.

The first problem we found was related to the spatial geometry of the model. Because each SU interacts only with its first neighbors, and veins act as water reservoirs with a constant $\Psi$, one areola was hydraulically isolated from the other. So, the results obtained for a leaf with, for instance, $100 \times 100$ SUs $(25 \times 25$ areolas), were exactly the same that would have been obtained by simulating an areola 625 times and arranging them side by side in a grid. So the conclusion presented in [5], that areolas synchronize if the initial conditions (and parameters) are the same, is easy to be understood and quite obvious indeed. In larger areolas no inner stomata opens, since there is never enough water coming into them from the veins. So, it is sufficient to study what happens with a single areola. For the sake of visualization, we will give most of the examples for areolas made of $4 \times 4$ SUs.

Regarding the time evolution of this model, we observed two important features.

1. The asymptotic behavior does not depend on initial conditions or on morphological details of each pore.

If one waits enough time for transients to die, each SU of an areola converges exactly to the same steady state conductance value. Differences in the morphology and initial conditions of SUs do not change this behavior, as can be seen in Figure 2. The number $(1,1),(1,2)$ and $(2,2)$ show the only three possible relative positions of the SU with respect to veins (see Fig. 1).

In Figure 2 (a) we show the time evolution of conductance $g^{i}$ for all $16 \mathrm{SU}$ of the areola when random values of $\theta^{i}$ are assigned to them, and in Figure 2 (b) time evolution of conductance $g^{i}$ when $\theta_{i}$ is the same, but initial conditions are not. One can see that the steady state behavior is not affected neither by the choice of mechanical coefficients nor by the initial conditions.

2. No sustained oscillations or patchiness are observed in the limit of continuous time evolution. Indeed, the time evolution of equation (1) was inspired in a differential equation,

$$
\frac{d \Psi}{d t} \approx \frac{\Delta \Psi}{\Delta t}=c\left(F^{i}-E^{i}\right),
$$

that is,

$$
\begin{gathered}
\Psi(t+1) \approx \Psi(t)+a\left(F^{i}-E^{i}\right), \\
\text { with } a=c \Delta t .
\end{gathered}
$$

This approximation is reasonable for small values of $\Delta t$ only. The parameter $a$ is the product of a constant $c$ (finite, with biological meaning of hydraulic conductivity) and $\Delta t$, which should be as small as possible. If a smaller value for $\Delta t$ is employed, the only change should be in the number of time steps needed to simulate the same total time. 


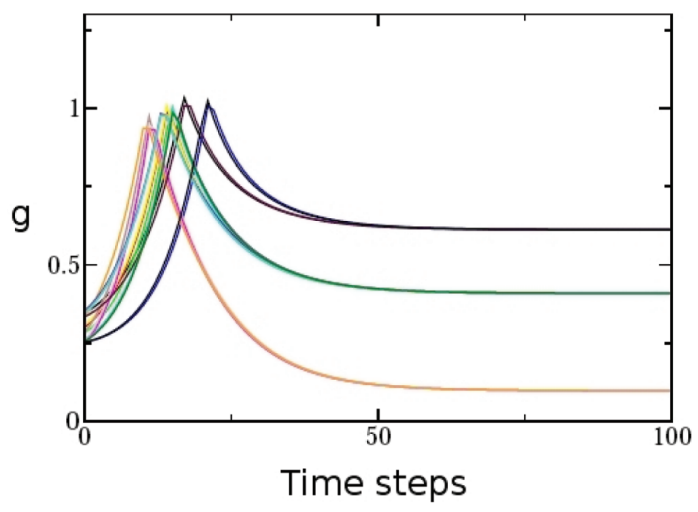

(a) Different mechanical coeficient.

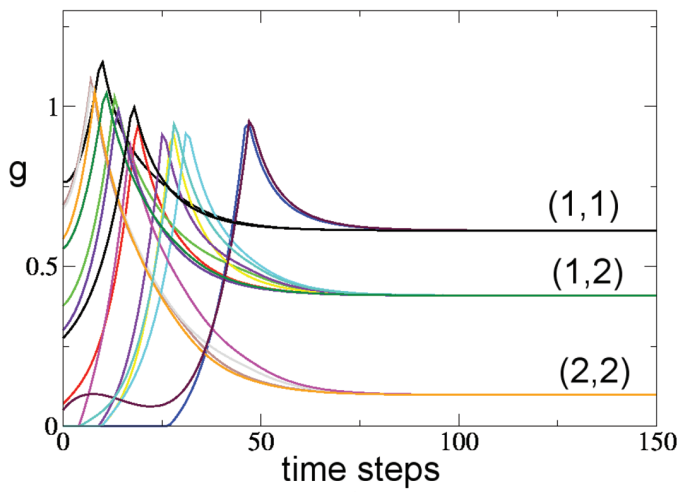

(b) Different initial conditions.

Figure 2 - Time evolution of individual conductance of all 16 SUs of an areola. (a) SUs have different mechanical coefficients; Bottom: SUs have same mechanical coefficients, but different initial conditions. $a=0.008, \alpha=0,1$ and $\Delta w=15, q=1,-3,0 \leq \pi_{g} \leq-1,5$ and $-2,0 \leq \Psi \leq 0$ in $t=0$.

This is equivalent to consider (except by a scaling factor) smaller and smaller values for $a$. On the other hand, if the value of $\Delta t$ (or $a$ ) is not small enough, the system apparently will never reach an stationary state, moving back and forth around the steady state value, in a behavior that could have been taken by real oscillations ${ }^{1}$. This is exactly what we have observed in our simulations: the aperture of each SU adjusted itself to balance the water fed into the system through the xylem (defined by its average distance from veins) with the water lost in the pores through evaporation. That is observed even if we consider a less restrictive geometry that circumvents the problem pointed out in the item above. So we think that maybe the oscillations reported by [5] were an artifact of discreteness of time. The time evolution of $\Psi(t)$ can not depend on the value of $\Delta t$ (the same reasoning can be applied to equation 4 and $\alpha$ ).

\section{A MODEL FOR HOMOBARIC LEAVES}

In view of these results, we decided to consider another geometry, in which the veins, or water sources, are randomly distributed over the leaf. The fraction $v$ of sites in the grid defined as veins is a new parameter and can be related to the water uptake from the soil through the xylem to the leaf. The veins form natural obstacles to the transport of water among neighboring SUs, but do not fully isolate them in areolas. Also, it seems more realistic to assume the existence of sources of water throughout the leaf, since the water available in the bulk of the leaf is also due to movements of molecules on wet cell walls. We adopted periodic boundary conditions, which means that we are considering a very large leaf, and looking at the dynamics in the bulk. In our simulations we observed that stomatal apertures did form spatial patches, but the aperture of each individual pore was still static: there were no moving patches or sustained oscillations. Figure 3 shows the time evolution of some SUs with different numbers of neighboring 'veins' in that case.

We then introduced modifications in the dynamics that controls opening and closing of stomata. We assumed that the opening movement was divided into two distinct phases [3, 6], a stress phase and a motor phase. According to these authors, during the stress phase the guard cell pressure appears to increase, but pores remain closed. At a threshold pressure level, the pores open slightly, commencing motor phase. Beyond this point, stomatal opening progresses rapidly. The stomata literally 'pops' open once a sufficient guard cell osmotic pressure potential has been achieved. This effect can be introduced if we replace equation 3 by (see Fig. 4).

$$
A^{i}=\left\{\begin{array}{l}
\frac{1}{2} \tanh \left[\gamma \Delta P-d_{i}\right] \quad \text { if } \Delta P>0 \\
\text { and } \Delta P(t)>\Delta P(t-1) \text { (opening) } \\
\frac{1}{2} \tanh \left[\gamma \Delta P-d_{0}\right] \quad \text { if } \Delta P>0 \\
\text { and } \Delta P(t)<\Delta P(t-1) \text { (closing) } \\
0 \text { if } \Delta P \leq 0
\end{array},(7)\right.
$$

\footnotetext{
${ }^{1}$ In eq. (2), if $a$ is not small enough, $\Psi(t+1)$ will apparently 'oscillate' (back and forth) around the steady state value $\Psi_{\infty}$, since $\left|a\left(F^{i}-E^{i}\right)\right|$ is larger than $\left|\Psi(t+1)-\Psi_{\infty}\right|$ This movement, due only to limitations of the numerical approximation, disappears if $a$ is small enough, and shall not be interpreted as real oscillations, that would not depend on the value of $a$. The same reasoning is applied to parameter $\alpha$.
} 
where $\tanh (x)$ is the hyperbolic tangent function, and $\Delta P=$ $P_{g}(t)-P_{e}(t)$ is the turgor pressure difference in time $t$; parameters $d_{0}$ and $d^{i}=d_{0}+c^{i}$ define the opening (closing) threshold. If $c^{i}=0$, opening and closing movements follow exactly the same path; if $c^{i}>0$ opening and closing follow different routes. Such properties seem to have been observed in the effects of water potential on xylem hydraulic resistance with growing experimental evidence of its importance to stomata control mechanisms [1]. We allow the parameter $c$ to vary from one SU to the other, to account for small differences in the morphology of each pore (the value of $d_{i}$ was randomly chosen in an interval $\left.\left[d_{0}, d_{\max }\right]\right)$.

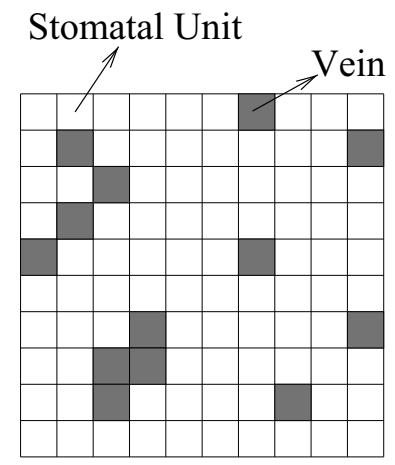

(a) New geometry, with a random distribution of veins.

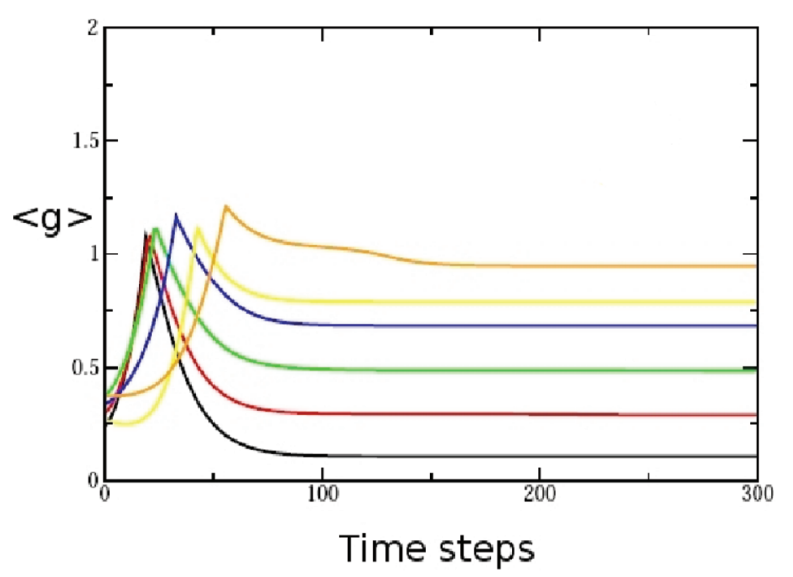

(b) Conductance of SUs with different number of veins in the neighborhood.

Figure 3 - Example of a leaf made of $10 \times 10$ SUs, in the model with random veins (a). Veins (in gray, with constant water potential) are now chosen at random, and periodic boundary conditions are adopted. The model is more adequate to describe homobaric leaves. (b) shows the time evolution of the conductance of six typical SUs. From top to bottom, the behavior of SUs with $0,1,2,3,4$ and 5 veins in the neighborhood; although there is spatial patchiness, the patches are static, since the conductance of every individual SU converges to a constant value, defined only by the position of the SU in respect to the distribution of veins. $\Delta w=15$, the fraction of veins $v=0.2$ and the leaf was made of $50 \times 50$ SUs.

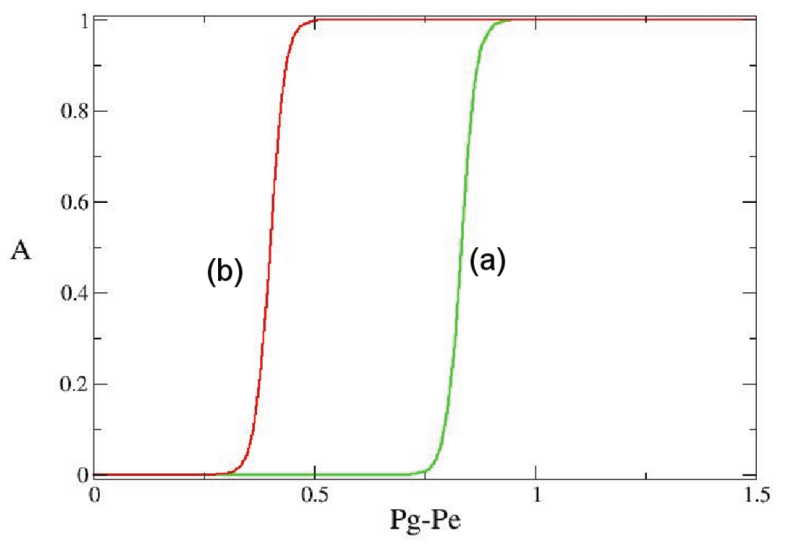

Figure 4 - Plot of equation (7), that gives the aperture $A$ as a function of the difference in turgor pressures of epidermis and guard cells, for $d_{0}=12, d_{i}=13$ and $\gamma=30$. Curve (a) is followed during opening movement and (b) when stomata is closing.

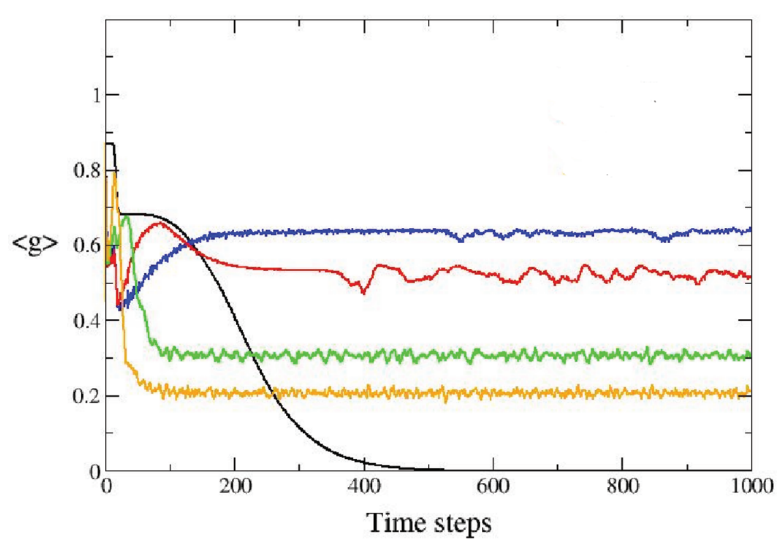

Figure $\mathbf{5}$ - Average conductance in a leaf for different values of water vapor mole fraction $\Delta w$. Note that, with the same parameters except for $\Delta w$, we observe different dynamical regimes. From top to bottom: $\Delta w=3,5,10,15$ and 0 ; $a=0,008, \alpha=0,1$.

If we consider equation (7) with $d_{i}=d_{0}$ (opening and closing follow the same route), the behavior of the system does not change qualitatively from what was observed with equation (3). The spatial patchiness of the steady state increases, but the patches are still constant in time. However, for $d_{i} \neq d_{0}$ we are able to observe, as a function of the relation between $\Delta w$ and $v$, steady oscillations, total closure or constant uniform behavior for the average conductance $<g>$. Also, with the graphical representation, it has been possible to observe patches with most of the qualitative features observed in experiments: The movements were somehow restricted by veins, but not confined to a fixed region; although evaporating sites closer to veins stayed more time opened than inner sites, the latter - some times very far from veins - opened widely from time to time, and not in an independent way, but in patches formed by synchronized SUs. Figure 5 shows the 
average conductance $<g>$ of the whole leaf, for different values of $\Delta w$ (and fixed $v$ ). We observe that the same model produces different dynamics, in different environmental conditions. For small values of the water vapor deficit, the conductance of the leaf converges to a constant value, but for higher values it is possible to observe oscillations.

\section{CONCLUSION}

In conclusion, we revisited a previous work [5], that presented a model for patchy behavior based on mechanisms of water transport in the mesophyll of the leaf, pointing out important limitations in it. Introducing two important modifications in the model, however, we showed that the mechanism of water transport from the xylem to the evaporating sites may still be sufficient to explain stomatal patchiness. Stomatal patchiness is observed in a wide variety of plants that, in general, display different behavior under different environmental or metabolic conditions. Our work suggests that some kind of inertia, recent memory, or else, the existence of some refractory period for the response of each individual pore to metabolic conditions in the surrounding tissue, may be enough to explain why stomata dynamics displays such a rich complex behavior. The hypothesis of hysteresis is very plausible and has been observed in other cell behaviors subjected to threshold dynamics, like neurons. We hope to stimulate further work to check the validity of this suggestion.

\section{ACKNOWLEDGMENTS}

The authors thank FAPESP (Fundação de Amparo a Pesquisa do Estado de São Paulo) and CNPq (Conselho Nacional de Pesquisa e Desenvolvimento Tecnológico) for financial support.

\section{REFERENCES}

[1] BUCKLEY TN. 2005. The control of stomata by water balance. New Phytol., 168: 275-292.

[2] BUCKLEY TN, MOTT KA \& FARQUHAR GD. 2003. A hydromechanical and biochemical model of stomatal conductance. Plant Cell Environ., 26: $1767-1785$.

[3] COOKE JR, DEBAERDEMAEKER JG, RAND RH \& MANG HA. 1976.
Finite-Element shell analysis of guard cell deformations. Transac. ASAE 19: $1107-1121$

[4] DELWICH MJ \& COOKE JR. 1977. An analytic model of the hydraulic aspects of stomatal dynamics. J. Theor. Biol., 69: 113-141.

[5] HAEFNER JW, BUCKLEY TN \& MOTT KA. 1997. A spatially explicit model of patchy stomatal responses to humidity. Plant Cell Environ., 20: 1087-1097.

[6] MEIDNER H \& EDWARDS M. 1975. Direct measurements of turgor pressure potentials of guard cells. J. Exp. Bot., 26: 319-330.

[7] MOTT KA \& BUCKLEY TN. 1998. Stomatal heterogeneity. J. Exp. Bot., 49: 407-417.

[8] MOTT KA \& BUCKLEY TN. 2000. Patchy stomatal conductance: emergent collective behavior of stomata. Trends Plant Sci., 5: 258-262.

[9] MOTT KA \& PEAK D. 2006. Stomatal patchiness and Task-performing Networks. Ann. Bot. 1-8, Advance Access published on November 3, 2006; doi:10.1093/aob/mcl234.

[10] PEAK D, WEST JD, MESSINGER SM \& MOTT KA. 2004. Evidence for complex, collective dynamics and emergent, distributed computation in plants. Proc. Nat. Acad. Sci. USA, 101: 918-922.

[11] PIERUSCHKA R, SCHURR U \& JAHNKE S. 2005. Lateral gas diffusion inside leaves, J. Exp. Bot. 56: 857-864.

[12] RAND RH \& ELLENSON JL. 1989. Dynamics of stomata fields in leaves. Planta, 177: 35-46.

[13] SAXE H. 1979. A structural and functional study of the co-ordinated reactions of individual Commelina communis L. stomata. Amer. J. Bot., 66: 1044-1052.

[14] SOUZA GM, OLIVEIRA RF \& CARDOSO VJM. 2004. Temporal dynamics of stomatal conductance of plants under water deficit: can homeostasis be improved by more complex dynamics? Braz. Arch. Biol. Tech., 47: 423-431.

[15] SOUZA GM, PINCUS SM \& MONTEIRO JAF. 2005. The complexitystability hypothesis in plant gas exchange under water deficit. Braz. J. Plant Physiol., 17: 363-373.

[16] SPENCE RD. 1987. The problem of variability in stomatal responses, particularly aperture variance, to environmental and experimental conditions. New Phytol., 107: 303-315. 